\title{
Radio Signals from Axion Dark Matter Conversion in Neutron Star Magnetospheres
}

\author{
Anson Hook, ${ }^{1, *}$ Yonatan Kahn, ${ }^{2,3,4, \dagger}$ Benjamin R. Safdi, ${ }^{5,}$ and Zhiquan Sun ${ }^{5, \S}$ \\ ${ }^{1}$ Department of Physics, Maryland Center for Fundamental Physics, University of Maryland, \\ College Park, Maryland 20742, USA \\ ${ }^{2}$ Department of Physics, Princeton University, Princeton, New Jersey 08544, USA \\ ${ }^{3}$ Kavli Institute for Cosmological Physics, University of Chicago, Chicago, Illinois 60637, USA \\ ${ }^{4}$ University of Illinois Urbana-Champaign, Urbana, Illinois 61801, USA \\ ${ }^{5}$ Department of Physics, Leinweber Center for Theoretical Physics, University of Michigan, Ann Arbor, Michigan 48109, USA
}

(Received 12 May 2018; revised manuscript received 13 August 2018; published 13 December 2018)

\begin{abstract}
We show that axion dark matter may be detectable through narrow radio lines emitted from neutron stars. Neutron star magnetospheres host both a strong magnetic field and a plasma frequency that increases towards the neutron star surface. As the axions pass through the magnetosphere, they can resonantly convert into radio photons when the plasma frequency matches the axion mass. We solve the axion-photon mixing equations, including a full treatment of the magnetized plasma, to obtain the conversion probability. We discuss possible neutron star targets and how they may probe the QCD-axion parameter space in the mass range of $\sim 0.2-40 \mu \mathrm{eV}$.
\end{abstract}

DOI: 10.1103/PhysRevLett.121.241102

The QCD axion is one of the best-motivated dark matter (DM) candidates: In addition to explaining the observed abundance of DM [1-3], the axion may also resolve the strong $C P$ problem [4-7]. However, testing the axion DM hypothesis requires probing extremely weak axion couplings. In particular, axions interact with photons through $\mathcal{L}_{a \gamma}=-g_{a \gamma \gamma} a \mathbf{E} \cdot \mathbf{B}$, where $g_{a \gamma \gamma} \sim 10^{-15} \mathrm{GeV}^{-1}$ for an axion of mass $m_{a}=10^{-6} \mathrm{eV}$. Direct detection experiments such as ADMX [8-10] are just beginning to probe small regions of the QCD-axion parameter space. In this Letter, we discuss a new avenue for testing the axion DM hypothesis. We show that in the presence of axion DM, monochromatic radio signals are emitted from neutron stars (NSs) through axion-photon conversion within the NS magnetosphere.

As first noted in Ref. [11], the finite electron density in the plasma within the NS magnetosphere gives the photon a mass $m_{\gamma}$, which modifies the photon dispersion relation and allows nonadiabatic resonant conversion of axions to photons. This photon mass is expected to fall off monotonically with distance from the NS surface. Thus, for any axion mass smaller than the plasma mass at the NS surface, there exists a conversion radius $r_{c}$ at which $m_{a}=m_{\gamma}$ and nonadiabatic resonant conversion occurs. However, the plasma around a NS is highly magnetized, with the cyclotron frequency $\Omega_{c}$ much larger than the plasma

Published by the American Physical Society under the terms of the Creative Commons Attribution 4.0 International license. Further distribution of this work must maintain attribution to the author(s) and the published article's title, journal citation, and DOI. Funded by SCOAP . frequency $\omega_{p}$, and the effective photon mass is anisotropic. In this Letter, we solve the axion-photon equations of motion within the NS magnetosphere to calculate the conversion probability and find that resonant conversion occurs over a distance $L \sim \sqrt{r_{c} v_{c} / m_{a}}$, where $v_{c}$ is the axion velocity at $r_{c}$.

Axion-photon conversion in neutron star magnetospheres is related to the detection mechanism utilized by axion helioscopes, such as CAST, to search for relativistic axions produced inside of the Sun which propagate to Earth [12-14]. Solar axions have a thermal spectrum $E \sim \mathrm{keV}$ and can convert to photons within CAST's static magnetic field $\mathbf{B}$. When the momentum transfer $q=$ $m_{a}^{2} /(2 E)$ is much less than the length of the $B$-field region $L(q L \ll 1)$, the conversion probability scales as $P_{a \gamma} \sim g_{a \gamma \gamma}^{2} \mathbf{B}^{2} L^{2}$. However, for large $m_{a}$ the conversion rate drops rapidly because the axion and photon have different dispersion relations, which becomes more apparent for larger values of $m_{a}$ and longer distances $L$. To circumvent this issue and maintain sensitivity to high- $m_{a}$ axions, CAST fills the $B$-field region with ${ }^{4} \mathrm{He}$ and ${ }^{3} \mathrm{He}$. By varying the pressure of the gas, the plasma frequency can be adjusted to give the photon a mass and match the axion dispersion over a range of $m_{a}$ values. With this technique, CAST has set some of the strongest limits in the mass range $m_{a} \sim 10^{-4}-10^{0} \mathrm{eV}[13,14]$.

NSs have long been recognized as promising targets for axion searches due to their strong magnetic fields. Previous efforts have focused on either photon-axion conversion leading to spectral distortions in the outgoing electromagnetic emission [15] or the conversion of thermal axions from the NS interior into photons in the magnetosphere 
[16]. However, neither of these processes require the axion to be $\mathrm{DM}$, nor are they sensitive enough to probe the QCD axion. In particular, thermal axions are ultrarelativistic and, hence, cannot undergo resonant conversion in the magnetosphere [17], but DM axions are only mildly relativistic and resonant conversion is obtained over a broad range of parameters, as we show. Reference [11] calculated the radio flux from DM axions infalling on NSs and converting into radio photons in the regime where the DM mixes strongly with the photon within the magnetosphere. Our work builds upon this previous work by calculating the radio flux from this process in the weak-mixing limit, applicable for QCDaxion-strength couplings $g_{\text {ary }}$. Additionally, we present a more precise calculation of the outgoing flux and show that current radio searches may be sensitive to QCD-axionstrength couplings from a variety of NS targets.

Most previous efforts to detect axion DM have focused on direct detection; see Ref. [18] for a detailed review. Though some experiments, such as CASPEr [19], exploit the axion coupling to nucleons, the majority of experiments aim to detect the coupling of the axion to electromagnetic fields. The combination of the ABRACADABRA [20,21], DM-Radio [22,23], ADMX [10,24], HAYSTAC [25-27], and MADMAX [28,29] experiments can potentially probe the QCD-axion coupling to photons over the wide mass range $m_{a} \sim 10^{-9}-10^{-4} \mathrm{eV}$. Our work complements these approaches by providing an avenue for indirect detection of QCD-axion DM in the mass range $\sim 0.2-40 \mu \mathrm{eV}$ utilizing existing and planned radio telescopes.

Neutron star magnetosphere.-The magnetic field in the vicinity of the NS surface is well described by a dipole configuration, with an axis $\hat{\mathbf{m}}$ that is misaligned from the rotation axis (which we take to be the $z$ axis) by an angle $\theta_{m}$. Charged particles are stripped from the surface of the NS at the magnetic poles and accelerated along open field lines, producing the nonthermal, pulsed radio, and gammaray emission seen from pulsars. These regions near the magnetic poles are characterized by a high-density, boosted plasma. On the other hand, the NS "lobes" consist of closed magnetic field lines and likely much more modest plasma densities. We will take a simplistic model for the neutron star magnetosphere inspired by Goldreich and Julian (GJ) [30], which gives the minimum plasma density in the presence of the NS magnetic field necessary for a selfconsistent solution to Maxwell's equations where particles on magnetic field lines corotate with the star.

Though originally proposed for aligned NSs with $\theta_{m}=0$, the GJ derivation applies equally well to misaligned NSs and gives a charge density

$$
n_{c}=\frac{2 \boldsymbol{\Omega} \cdot \mathbf{B}}{e} \frac{1}{1-\Omega^{2} r^{2} \sin ^{2} \theta},
$$

where $\Omega=2 \pi / P$ with $P$ the NS spin period, and $\theta$ is the polar angle with respect to the rotation axis. We will take the charge density as a rough estimate of the electron number density: $n_{e}=\left|n_{c}\right|$. The plasma frequency is $\omega_{p} \approx \sqrt{4 \pi \alpha n_{e} / m_{e}}$, so that within the GJ model

$$
\omega_{p} \approx\left(1.5 \times 10^{2} \mathrm{GHz}\right) \sqrt{\left(\frac{B_{z}(r)}{10^{14} \mathrm{G}}\right)\left(\frac{1 \mathrm{sec}}{P}\right)},
$$

where $B_{z}=\left(B_{0} / 2\right)\left(r_{0} / r\right)^{3}\left[3 \cos \theta \hat{\mathbf{m}} \cdot \hat{\mathbf{r}}-\cos \theta_{m}\right]$ is the component of the magnetic field along the $\hat{\mathbf{z}}$ direction. Note that $\hat{\mathbf{m}} \cdot \hat{\mathbf{r}}=\cos \theta_{m} \cos \theta+\sin \theta_{m} \sin \theta \cos (\Omega t)$ depends on time due to the rotation of the NS. In Eq. (2), we have neglected the relativistic correction in the denominator of Eq. (1), which can be important for millisecond pulsars but is typically a percent-level correction for large $P$. In practice, the true plasma density is likely more complicated than the simple GJ model, with possibly nontrivial time dependence and boosts. However, the GJ model provides a straightforward starting point for this analysis, which we hope can be improved in future work. In this analysis, we focus only on the region of closed field lines where the plasma is expected to be nonrelativistic, leaving the complications of boosted plasma near the magnetic poles to future work.

As we show below, the axion-photon conversion occurs resonantly within the vicinity of the conversion radius $r_{c}$ defined to be the radius at which $\omega_{p}=m_{a}$. Using the expressions above, we find that in the GJ model,

$$
\begin{aligned}
r_{c}\left(\theta, \theta_{m}, t\right)= & 224 \mathrm{~km} \times\left|3 \cos \theta \hat{\mathbf{m}} \cdot \hat{\mathbf{r}}-\cos \theta_{m}\right|^{1 / 3} \\
& \times\left(\frac{r_{0}}{10 \mathrm{~km}}\right) \times\left[\frac{B_{0}}{10^{14} \mathrm{G}} \frac{1 \mathrm{sec}}{P}\left(\frac{1 \mathrm{GHz}}{m_{a}}\right)^{2}\right]^{1 / 3} .
\end{aligned}
$$

Photon flux from mixing equations.-Since the axion DM starts out nonrelativistic far away from the NS and is accelerated to semirelativistic velocities at radius $r_{c}$, we can approximate the axion trajectories as radial. In the Supplemental Material [31], we give a set of physical arguments that may be used to understand the parametric dependence of the axion-photon conversion probability. Here, we focus on the axion-photon conversion probability and the varying photon speed, which are important factors in calculating the outgoing photon flux. The combination of these two effects gives the energy flux at infinity sourced by a given infalling axion DM density, which we refer to as the energy transfer function. Numerically, both of these effects are captured simultaneously by solving the axionphoton coupled differential equation. In analogy with standard nonadiabatic conversion [36,37], we calculate the energy transfer function by solving the coupled wave equations for the axion-photon system in the presence of the interaction term $-g_{a \gamma \gamma} a \mathbf{E} \cdot \mathbf{B}$ in the Lagrangian, which induces mixing between the axion $a$ and the component of the photon vector potential $A_{\|}$transverse to the axion's motion but coplanar with the magnetic field. We then show 
that because the initial photons are produced at nonrelativistic velocities, the final outgoing flux is velocity suppressed.

Following Ref. [17], we assume radial plane wave solutions of the form $a(r, t)=i e^{i \omega t-i k r} \tilde{a}(r)$ and $A_{\|}(r, t)=$ $e^{i \omega t-i k r} \tilde{A}_{\|}(r)$, where $k^{2}=\omega^{2}-m_{a}^{2}$. As we will show, the resonant conversion takes place in a narrow enough region around $r_{c}$ that we may neglect the $r$ dependence of $\omega$ due to gravitational effects. Similarly, while the dispersion relation for $k$ holds for both the axion and the photon at $r_{c}$, the photon dispersion changes away from the conversion radius due to the continuously varying plasma mass. We account for both of these effects in turn. The analytic arguments presented below are supported by a full numerical analysis in the Supplemental Material [31], where we also derive the equations of motion for the coupled axion-photon system in the plasma.

Near $r_{c}$, we may use the WKB approximation $\left|\tilde{A}_{\|}^{\prime \prime}(r)\right| \ll$ $k\left|\tilde{A}^{\prime}(r)\right|$ and $\left|\tilde{a}^{\prime \prime}(r)\right| \ll k\left|a^{\prime}(r)\right|$. The mixing equations reduce to the first-order ordinary differential equation

$$
\left[-i \frac{d}{d r}+\frac{1}{2 k}\left(\begin{array}{cc}
m_{a}^{2}-\xi \omega_{p}^{2} & \Delta_{B} \\
\Delta_{B} & 0
\end{array}\right)\right]\left(\begin{array}{c}
\tilde{A}_{\|} \\
\tilde{a}
\end{array}\right)=0,
$$

where

$$
\xi=\frac{\sin ^{2} \tilde{\theta}}{1-\frac{\omega_{p}^{2}}{\omega^{2}} \cos ^{2} \tilde{\theta}}, \quad \Delta_{B}=B g_{a \gamma \gamma} m_{a} \frac{\xi}{\sin \tilde{\theta}} .
$$

In these expressions, $\omega=m_{a} \sqrt{1+v_{c}^{2}}$ and $k=m_{a} v_{c}$, with $v_{c}$ the DM velocity at $r=r_{c}$. We have also defined $\tilde{\theta}$ as the angle between the propagation direction $\hat{\mathbf{r}}$ and the magnetic field $\mathbf{B}$.

For $r \gg r_{c}$, the axion-photon system no longer strongly mixes, but the amplitude of $A_{\|}$modulates due to the varying plasma frequency of the medium. This effect is exactly analogous to the increasing amplitude of ocean waves as they approach the shore (though in our analysis, we are considering the opposite case of waves leaving the shore). The net effect is a suppression of the outgoing electromagnetic wave by a factor of $\sqrt{v_{c}}$ asymptotically far away from the NS: $A_{\|}(\infty) \sim \sqrt{v_{c}} A_{\|}\left(r_{c}\right)$. From the particle point of view, this decrease in the electromagnetic energy density is simply particle number conservation. As the velocity increases towards the speed of light, the requirement that the initial energy flux equals the final energy flux dictates that the final outgoing energy density is suppressed by a factor of $v_{c}$.

To calculate the electromagnetic flux asymptotically far from the NS, we proceed by analogy to time-dependent perturbation theory in the Schrödinger equation, working to first order in $\Delta_{B}$ [17]. Taking initial conditions $\tilde{A}_{\|}\left(r_{0}\right)=0$ and $\tilde{a}\left(r_{0}\right)=a_{0}$ and neglecting the modulation of the outgoing electromagnetic wave for now, Eq. (4) implies

$$
\begin{aligned}
F_{a \gamma}(r) \equiv & \frac{\left|A_{\|}(r)\right|^{2}}{a_{0}^{2}} \\
= & \mid \int_{0}^{r} d r^{\prime} \frac{B\left(r^{\prime}\right) \xi\left(r^{\prime}\right) g_{a \gamma \gamma}}{2 v_{c} \sin \tilde{\theta}} \\
& \times\left. e^{\left\{\left[-i \int_{0}^{r^{\prime}} d \tilde{r}\left(m_{a}^{2}-\xi(\tilde{r}) \omega_{p}^{2}(\tilde{r})\right] /\left[2 m_{a} v_{c}\right]\right\}\right.}\right|^{2} .
\end{aligned}
$$

Taking $r \rightarrow \infty$ and including the amplitude modulation of the outgoing electromagnetic field, we evaluate Eq. (6) by the method of stationary phase to obtain the energy transfer function

$$
p_{a \gamma}^{\infty} \equiv v_{c} \lim _{r \rightarrow \infty} F_{a \gamma}(r) \approx \frac{1}{2 v_{c}} g_{a \gamma \gamma}^{2} B\left(r_{c}\right)^{2} L^{2},
$$

where $L=\sqrt{2 \pi r_{c} v_{c} /\left(3 m_{a}\right)}$ may be interpreted as the distance over which the resonant conversion takes place at $\tilde{\theta}=\pi / 2$. However, Eq. (7) holds for generic $\tilde{\theta}$ to leading order in $v_{c}$. Note that Eq. (7) only applies in the limit of sufficiently small $g_{a \gamma \gamma}$ and $B\left(r_{c}\right)$. For larger parameter values, one should take the solution from the full first-order differential Eq. (4) or the second-order differential equation detailed in the Supplemental Material [31].

Radiated power.-Next, we calculate the electromagnetic power emitted from the NS by axions converting into photons. Since the NS plasma is optically thin, Thomson scattering of photons is negligible for the long-period NSs under consideration [17] and, thus, outgoing photons do not scatter. Because $m_{a}^{-1} \ll r_{c}$ for $m_{a}$ in the MHz-GHz range, $L$ is parametrically smaller than $r_{c}$ and, thus, conversion takes place in a small region around $r_{c}$. We thus estimate the radiated power $\mathcal{P}$ by multiplying the flux of DM through a surface subtending a solid angle $d \Omega$ at $r_{c}$ by the energy transfer function:

$$
\frac{d \mathcal{P}\left(\theta, \theta_{m} t\right)}{d \Omega} \approx 2 \times p_{a \gamma}^{\infty} \rho_{\mathrm{DM}}^{r_{c}} v_{c} r_{c}^{2},
$$

where $\rho_{\mathrm{DM}}^{r_{c}}$ is the DM mass density at $r=r_{c}$. All quantities on the right-hand side of Eq. (8) depend on $\theta, \theta_{m}$, and $t$ through their dependence on $r_{c}$ [see Eq. (3)]. The factor of 2 comes from the fact that the DM may convert into photons either on its way into the NS or out of the NS; if it is converted on the way in, then the photon is reflected back out, since the higher-density plasma acts as a mirror to photons of frequency $\omega<\omega_{p}$.

Let us assume that asymptotically far away from the NS, the DM has density $\rho_{\mathrm{DM}}^{\infty}$ and is described by a MaxwellBoltzmann velocity distribution with

$$
f_{\infty}\left(\mathbf{v}_{\infty}\right)=\frac{1}{\pi^{3 / 2} v_{0}^{3}} e^{\left[\left(-\mathbf{v}_{\infty}^{2}\right) /\left(v_{0}^{2}\right)\right]},
$$

where $v_{0} \sim 10^{-3}$ is the DM virial velocity. We can calculate $v_{c}$ by conservation of energy: $v_{c}^{2}=v_{\infty}^{2}+\left[\left(2 G M_{\mathrm{NS}}\right) / r_{c}\right] \approx$ $\left[\left(2 G M_{\mathrm{NS}}\right) / r_{c}\right]$, where $v_{\infty}$ is the DM speed asymptotically 
far away from the NS, which is typically much smaller than the escape velocity $\sqrt{2 G M_{\mathrm{NS}} / r_{c}}$. Liouville's theorem maps the phase-space distribution from asymptotic infinity to $r_{c}: \rho_{\mathrm{DM}}^{r_{c}} f_{r_{c}}(\mathbf{v})=\rho_{\mathrm{DM}}^{\infty} f_{\infty}\left(\mathbf{v}_{\infty}[\mathbf{v}]\right)$, where $\mathbf{v}_{\infty}[\mathbf{v}]$ denotes the velocity at asymptotic infinity that gives velocity $\mathbf{v}$ at radius $r_{c}$. Integrating and expanding in the limit $v_{0}^{2} /\left(G M_{\mathrm{NS}} / r_{c}\right) \ll 1$ gives

$$
\rho_{\mathrm{DM}}^{r_{c}}=\rho_{\mathrm{DM}}^{\infty} \frac{2}{\sqrt{\pi}} \frac{1}{v_{0}} \sqrt{\frac{2 G M_{\mathrm{NS}}}{r_{c}}}+\cdots .
$$

This then leads to an expression for the radiated power:

$$
\begin{aligned}
& \frac{d \mathcal{P}\left(\theta=\frac{\pi}{2}, \theta_{m}=0\right)}{d \Omega}
\end{aligned}
$$

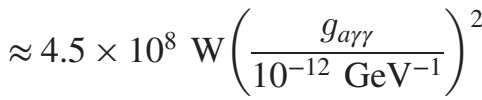

$$
\begin{aligned}
& \times\left(\frac{r_{0}}{10 \mathrm{~km}}\right)^{2}\left(\frac{m_{a}}{1 \mathrm{GHz}}\right)^{5 / 3}\left(\frac{B_{0}}{10^{14} \mathrm{G}}\right)^{2 / 3}\left(\frac{P}{1 \mathrm{sec}}\right)^{4 / 3} \\
& \times\left(\frac{\rho_{\infty}}{0.3 \mathrm{GeV} / \mathrm{cm}^{3}}\right)\left(\frac{M_{\mathrm{NS}}}{1 M_{\odot}}\right)\left(\frac{200 \mathrm{~km} / \mathrm{s}}{v_{0}}\right),
\end{aligned}
$$

with

$$
\begin{aligned}
\frac{d \mathcal{P}\left(\theta, \theta_{m}, t\right)}{d \Omega}= & \frac{d \mathcal{P}\left(\theta=\frac{\pi}{2}, \theta_{m}=0\right)}{d \Omega} \\
& \times \frac{3(\hat{\mathbf{m}} \cdot \hat{\mathbf{r}})^{2}+1}{\left|3 \cos \theta \hat{\mathbf{m}} \cdot \hat{\mathbf{r}}-\cos \theta_{m}\right|^{4 / 3}} .
\end{aligned}
$$

Both Eqs. (11) and (12) are formally only valid so long as $r_{c}>r_{0}$; no resonant conversion takes place inside the NS. This regulates the otherwise-divergent denominator in Eq. (12) and also gives a strong angular and time dependence to the signal.

Radio telescope sensitivity.-The radio flux at Earth is given by $F\left(\theta, \theta_{m}, t\right)=d \mathcal{P}\left(\theta, \theta_{m}, t\right) / d \Omega / d^{2}$, where $d$ is the distance from us to the NS. The figure of merit for radio telescopes is the flux density $S=F / B$, where $B$ is the bandwidth. Energy conservation implies that the expected bandwidth of the signal is $B \sim\left(v_{0} / c\right)^{2} m_{a} /(2 \pi)$ : The kinetic energy gained by the infalling DM is exactly canceled by the gravitational redshift of the outgoing photon, and the bandwidth of the signal is determined by the velocity dispersion in the asymptotic DM distribution. This argument does not hold exactly because the finite angular velocity of the magnetosphere leads to nonconservation of energy from the point of view of the outgoing photon. However, as derived in the Supplemental Material [31], this has a negligible effect on the bandwidth. We note also that the central frequency of the signal will be shifted by the velocity of an individual source with respect to Earth, which will not change the flux density but must be accounted for in extracting the axion mass from an observed signal.
The flux density is then

$$
\begin{aligned}
S= & 6.7 \times 10^{-5} \mathrm{Jy}\left(\frac{100 \mathrm{pc}}{d}\right)^{2}\left(\frac{1 \mathrm{GHz}}{m_{a}}\right) \\
& \times\left(\frac{200 \mathrm{~km} / \mathrm{s}}{v_{0}}\right)^{2}\left(\frac{d \mathcal{P} / d \Omega}{4.5 \times 10^{8} \mathrm{~W}}\right) .
\end{aligned}
$$

This should be compared to the minimum detectable flux at a radio telescope, which is given by

$$
S_{\min }=\mathrm{SNR}_{\min } \frac{\mathrm{SEFD}}{\sqrt{n_{\mathrm{pol}} B \Delta t_{\mathrm{obs}}}},
$$

where $\mathrm{SNR}_{\min }$ is the minimum signal-to-noise ratio, $\mathrm{SEFD}$ is the system-equivalent flux density, $n_{\text {pol }}$ is the number of polarizations (we will take $n_{\mathrm{pol}}=2$ ), $B$ is the bandwidth, and $\Delta t_{\mathrm{obs}}$ is the observation time. As an example, the Arecibo Telescope has SEFD 2 Jy. Equation (13) holds for sources whose bandwidth is wider than the intrinsic frequency resolution of the telescope, which we will assume is always the case. In the Supplemental Material [31], we discuss the optimization of the bandwidth for axion DM radio signals and how to account for the nontrivial time dependence of the light curve in our sensitivity estimates.

Neutron star targets. - As pointed out in Ref. [11], nearby isolated neutron stars (INSs) make excellent targets for radio signals from axion DM conversion. This group of approximately seven NSs is characterized by their proximity to Earth $(\lesssim 500 \mathrm{pc})$, strong magnetic fields $\left(\sim 10^{13} \mathrm{G}\right)$, long spin periods $(\sim 5 \mathrm{~s})$, and lack of observed pulsed, nonthermal emission (see, e.g., Refs. [38-40]). Importantly, since these NSs do not exhibit radio emission, we can estimate the sensitivity to axion DM assuming that we are limited by thermal noise in the radio telescope rather than background radiation from the NS. Additionally, the lack of nonthermal emission suggests that pair production at the NS surface is inefficient and that the GJ model for the plasma density may hold throughout the magnetosphere [11].

Figure 1 shows the sensitivity in $g_{a \gamma \gamma}$ from $100 \mathrm{~h}$ of observation of one of the isolated NSs, J0806.4-4123. This NS has a period $P \approx 11.37 \mathrm{~s}$, magnetic field $B_{0} \approx 2.5 \times 10^{13} \mathrm{G}$, and is at a distance $d \approx 250 \mathrm{pc}$ from Earth [40]. We also assume $M_{\mathrm{NS}}=1 M_{\odot}$ and $r_{0}=10 \mathrm{~km}$. We take SEFD $\approx 2 \mathrm{Jy}$ for our estimates, though this may be improved with future instruments such as the Square Kilometre Array. Our sensitivity curves are defined by $1 \sigma$ significance, as discussed in the Supplemental Material [31]. We show sensitivities calculated for two pulsar geometries. The solid curve takes a generic value $\theta=90^{\circ}$ for the polar angle of Earth in the NS frame, while the dashed curve is tuned to $\theta=120.5^{\circ}$, which gives the near maximal signal at low masses and which is also highly pulsed from emission at orientations where $r_{c} \rightarrow r_{0}$. In both cases, we take a generic misalignment angle $\theta_{m}=10^{\circ}$. The low-mass cutoff is set to the $m_{a} /(2 \pi)=50 \mathrm{MHz}$ threshold of typical radio telescopes, while the high-mass 


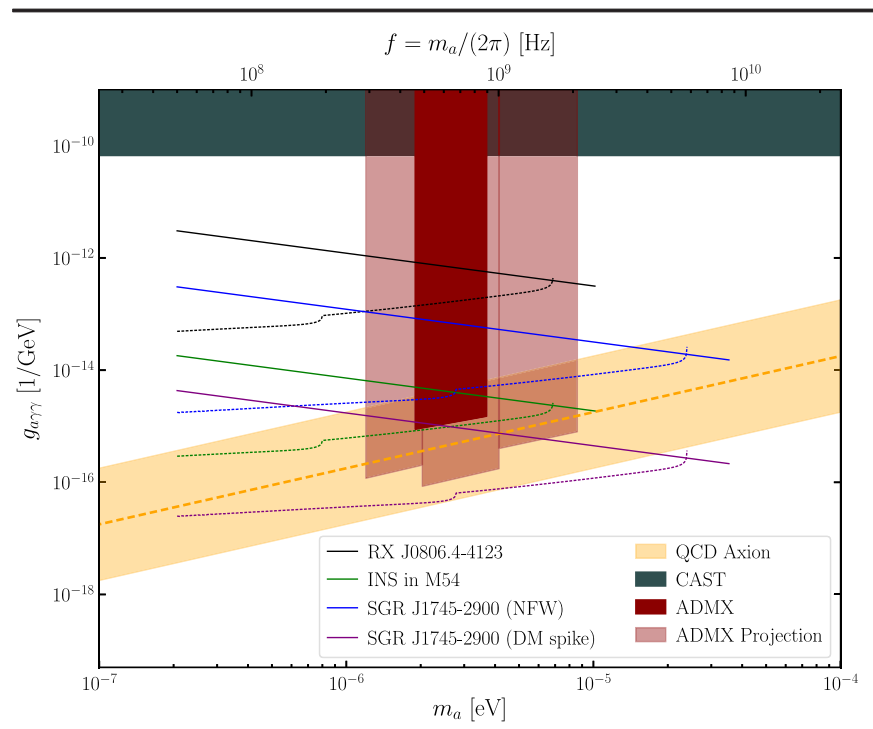

FIG. 1. Projected sensitivity to $g_{\text {ary }}$ as a function of axion mass $m_{a}$ for $\Delta t_{\mathrm{obs}}=100 \mathrm{~h}$ and SEFD $=2 \mathrm{Jy}$, alongside current and projected limits from ADMX and CAST. The QCD axion is predicted to lie within the orange band. We have taken $\theta_{m}=10^{\circ}$ and the solid (dashed) curves assume $\theta=90^{\circ}\left(\theta=120.5^{\circ}\right)$. The lower mass cutoff is set by the lowest available frequency of current radio telescopes, while the high-mass cutoff comes from requiring the conversion radius to be outside the NS radius.

cutoff is determined by the maximum mass for which the conversion radius is outside the NS radius.

We may also consider NSs in regions of high DM density and/or low velocity dispersion. For example, the magnetar SGR J1745-2900 is located $R \approx 0.1$ pc away from the Galactic Center [41-44], where the DM density is highly uncertain but could be significantly enhanced compared to the local density. Using the NFW and Burkert fits from Ref. [45], we find that the DM density at $R=0.1 \mathrm{pc}$ is enhanced by a factor of $2 \times 10^{5}$ for the best-fit NFW profile, relative to the local density, but only a factor $\sim 4$ for the best-fit cored Burkert profile, though the cored profiles may be in tension with new data from the Galactic bulge $[46,47]$. If the DM distribution were described by a generalized NFW profile with an index $\gamma=1.5$, which is allowed by the kinematic data available, then the enhancement would be $\sim 10^{7}$; if there is a DM density spike near Sgr $\mathrm{A}^{*}$, the supermassive black hole at the center of the Galaxy, the enhancement could be as large as $\sim 10^{9}$ [48].

Figure 1 shows the projected sensitivity from $100 \mathrm{~h}$ observation of SGR J1745-2900, assuming both the best-fit NFW DM profile (blue) and spike profile (purple), for $\theta=90^{\circ}$ (solid) and $\theta=120.5^{\circ}$ (dashed). We take $v_{0}=$ $200 \mathrm{~km} / \mathrm{s}$ and $d=8.5 \mathrm{kpc}$ for the distance to the Galactic Center and assume $M_{\mathrm{NS}}=1 M_{\odot}$ and $r_{0}=10 \mathrm{~km}$ as before, and use the inferred magnetic field $B_{0} \approx 1.6 \times 10^{14} \mathrm{G}$ and period $P \sim 3.76 \mathrm{~s}[41,42]$. Despite the fact that pulsed radio emission has been observed from this magnetar [43,44], we have made these sensitivity estimates under the assumption that the dominant noise source is the thermal noise in the telescope. Since the nonthermal radio emission is pulsed, nonpulsed (or pulsed but out of phase) DM-induced flux would likely still be dominated by telescope noise. Interestingly, as seen in Fig. 1, observations of SGR J1745-2900 could be sensitive to the QCD axion over multiple orders of magnitude in $m_{a}$, depending on the DM density profile. However, we stress that this sensitivity estimate relies on the GJ model, which may not apply to this magnetar.

Alternatively, one could consider isolated NSs within dwarf galaxies. In the Sagittarius Dwarf, the central DM density is enhanced by a factor $\sim 5 \times 10^{5}$ compared to the local density, and the velocity dispersion is low, $v_{0} \sim$ $10 \mathrm{~km} / \mathrm{s}$ [49]. The globular cluster M54 appears to be coincident with the center of the Sagittarius Dwarf Galaxy, with the cluster having a core radius $\sim 1 \mathrm{pc}$, a mass $\sim 2 \times 10^{6} M_{\odot}$, and a distance of around $\sim 20 \mathrm{kpc}$ from Earth [50,51]. Given the mass of M54, there are likely many hundreds of NSs within the central core [52]. Assuming that just one of these NSs has the properties of J0806.4-4123 gives the green curves shown in Fig. 1 (labeled INS in M54) for the two representative values of $\theta$. If there are $N$ such INSs in the field of view, then we may expect the sensitivity to improve as $1 / \sqrt{N}$. The fact that all NSs radiate at the same frequency from axion DM could make even more distant galaxies promising targets. On the other hand, due to the peculiar velocities of the individual NSs, the source signal will either be a forest of lines with relative bandwidths $\sim v_{0}^{2}$ or a broader line with relative bandwidth $\sim v_{0}$; the trade-off between source density and bandwidth is explored further in Ref. [53].

A narrow radio line from a NS target could provide a striking signature of axion DM. On the other hand, in the absence of a signal, it will be difficult to set a robust limit on $g_{\text {ary }}$ because of challenges in understanding confidently the plasma density and time-dependent dynamics in the inner regions of the magnetosphere. Towards that end, it would be useful to incorporate the physics of axion-photon conversion into NS simulations [54]. Such work should lead to more precise predictions for the radio-line signal.

Y. K. and B. S. thank Jesse Thaler for collaboration in the early stages of this project. We thank Anatoly Spitkovsky for detailed discussions regarding NS magnetospheres, and Nahum Arav, Kfir Blum, Junwu Huang, Paul Ray, Nicholas Rodd, Jonathan Squire, Christoph Weniger, and Kathryn Zurek for useful discussions. The work of B. R. S. and Z. S. was supported in part by the DOE Early Career Grant No. de-sc0019225.

Note added.-Recently, Ref. [55] appeared, which addresses similar questions. Our work differs in several respects, but importantly, where we do overlap, we disagree in detail with their results for the conversion probability, radio flux, and projected sensitivity. Specifically, Ref. [55] considers adiabatic resonant conversion, while we use nonadiabatic 
resonant conversion, and Ref. [55] claims (without detailed justification) that the signal bandwidth is $\mathcal{O}\left(v_{0}\right)$ from "Doppler broadening." While this is indeed true for an incoherent sum of signals from several sources [53], our energy conservation arguments strongly support the fact that the signal bandwidth from a single source must match the axion bandwidth at infinity, which is $\mathcal{O}\left(v_{0}^{2}\right)$.

*hook@umd.edu

†ykahn@uchicago.edu

*bsafdi@umich.edu

§qusun@umich.edu

[1] J. Preskill, M. B. Wise, and F. Wilczek, Cosmology of the invisible axion, Phys. Lett. 120B, 127 (1983).

[2] L. F. Abbott and P. Sikivie, A cosmological bound on the invisible axion, Phys. Lett. 120B, 133 (1983).

[3] M. Dine and W. Fischler, The not so harmless axion, Phys. Lett. 120B, 137 (1983).

[4] R. D. Peccei and H.R. Quinn, $C P$ Conservation in the Presence of Instantons, Phys. Rev. Lett. 38, 1440 (1977).

[5] R. D. Peccei and H. R. Quinn, Constraints imposed by $C P$ conservation in the presence of instantons, Phys. Rev. D 16, 1791 (1977).

[6] S. Weinberg, A New Light Boson?, Phys. Rev. Lett. 40, 223 (1978).

[7] F. Wilczek, Problem of Strong $P$ and $T$ Invariance in the Presence of Instantons, Phys. Rev. Lett. 40, 279 (1978).

[8] S. J. Asztalos et al. (ADMX Collaboration), Large scale microwave cavity search for dark matter axions, Phys. Rev. D 64, 092003 (2001).

[9] S. J. Asztalos, G. Carosi, C. Hagmann, D. Kinion, K. van Bibber, M. Hotz, L. J Rosenberg, G. Rybka, J. Hoskins, J. Hwang, P. Sikivie, D. B. Tanner, R. Bradley, and J. Clarke, SQUID-Based Microwave Cavity Search for Dark-Matter Axions, Phys. Rev. Lett. 104, 041301 (2010).

[10] T. M. Shokair et al., Future directions in the microwave cavity search for dark matter axions, Int. J. Mod. Phys. A 29, 1443004 (2014).

[11] M. S. Pshirkov and S. B. Popov, Conversion of dark matter axions to photons in magnetospheres of neutron stars, J. Exp. Theor. Phys. 108, 384 (2009).

[12] V. Anastassopoulos et al. (CAST Collaboration), New CAST limit on the axion-photon interaction, Nat. Phys. 13, 584 (2017).

[13] M. Arik et al. (CAST Collaboration), Search for Solar Axions by the CERN Axion Solar Telescope with ${ }^{3} \mathrm{He}$ Buffer Gas: Closing the Hot Dark Matter Gap, Phys. Rev. Lett. 112, 091302 (2014).

[14] M. Arik et al. (CAST Collaboration), New solar axion search using the CERN axion solar telescope with ${ }^{4} \mathrm{He}$ filling, Phys. Rev. D 92, 021101 (2015).

[15] D. Chelouche, R. Rabadan, S. Pavlov, and F. Castejon, Spectral signatures of photon-particle oscillations from celestial objects, Astrophys. J. Suppl. Ser. 180, 1 (2009).

[16] D. E. Morris, Axion mass limits from pulsar x rays, Phys. Rev. D 34, 843 (1986).
[17] G. Raffelt and L. Stodolsky, Mixing of the photon with low mass particles, Phys. Rev. D 37, 1237 (1988).

[18] I. G. Irastorza and J. Redondo, New experimental approaches in the search for axion-like particles, Prog. Part. Nucl. Phys. 102, 89 (2018).

[19] D. Budker, P. W. Graham, M. Ledbetter, S. Rajendran, and A. O. Sushkov, Proposal for a Cosmic Axion Spin Precession Experiment (CASPEr), Phys. Rev. X 4, 021030 (2014).

[20] Y. Kahn, B. R. Safdi, and J. Thaler, Broadband and Resonant Approaches to Axion Dark Matter Detection, Phys. Rev. Lett. 117, 141801 (2016).

[21] J. W. Foster, N. L. Rodd, and B. R. Safdi, Revealing the dark matter halo with axion direct detection, Phys. Rev. D 97, 123006 (2018).

[22] S. Chaudhuri, P. W. Graham, K. Irwin, J. Mardon, S. Rajendran, and Y. Zhao, Radio for hidden-photon dark matter detection, Phys. Rev. D 92, 075012 (2015).

[23] M. Silva-Feaver et al., Design overview of the DM radio pathfinder experiment, IEEE Trans. Appl. Supercond. 27, 1400204 (2017).

[24] L. J. Rosenberg, Dark-matter QCD-axion searches, Proc. Natl. Acad. Sci. U. S. A. 112, 12278 (2015).

[25] B. M. Brubaker et al., First Results from a Microwave Cavity Axion Search at $24 \mu \mathrm{eV}$, Phys. Rev. Lett. 118, 061302 (2017).

[26] S. Al Kenany et al., Design and operational experience of a microwave cavity axion detector for the $20-100 \mu \mathrm{eV}$ range, Nucl. Instrum. Methods Phys. Res., Sect. A 854, 11 (2017).

[27] B. M. Brubaker, L. Zhong, S. K. Lamoreaux, K. W. Lehnert, and K. A. van Bibber, The HAYSTAC axion search analysis procedure, Phys. Rev. D 96, 123008 (2017).

[28] B. Majorovits and J. Redondo (MADMAX Working Group), MADMAX: A new dark matter axion search using a dielectric haloscope, arXiv:1611.04549.

[29] A. Caldwell, G. Dvali, B. Majorovits, A. Millar, G. Raffelt, J. Redondo, O. Reimann, F. Simon, and Frank Steffen (MADMAX Working Group), Dielectric Haloscopes: A New Way to Detect Axion Dark Matter, Phys. Rev. Lett. 118, 091801 (2017).

[30] P. Goldreich and W. H. Julian, Pulsar electrodynamics, Astrophys. J. 157, 869 (1969).

[31] See Supplemental Material at http://link.aps.org/ supplemental/10.1103/PhysRevLett.121.241102 for further details and cross-checks concerning the calculation of the radiated flux, bandwidth, light-curve, and polarization of the signal, which includes Ref. [32-35].

[32] A. V. Gurevich, V. S. Beskin, J. N. Istomin, and Y. N. Istomin, Physics of the Pulsar Magnetosphere (Cambridge University Press, Cambridge, England, 2006).

[33] G. Cowan, K. Cranmer, E. Gross, and O. Vitells, Asymptotic formulae for likelihood-based tests of new physics, Eur. Phys. J. C 71, 1554 (2011); Erratum, Eur. Phys. J. C 73, 2501(E) (2013).

[34] W. Pauli, Theory of Relativity (Pergamon Press, New York, 1958).

[35] K. Tanaka, Reflection and transmission of electromagnetic waves by a linearly accelerated dielectric slab, Phys. Rev. A 25, 385 (1982). 
[36] L. D. Landau, Zur theorie der energieubertragung ii, Z. Sowjetunion 2, 46 (1932).

[37] C. Zener, Non-adiabatic crossing of energy levels, Proc. R. Soc. A 137, 696 (1932).

[38] W. Voges, B. Aschenbach, T. Boller, H. Bräuninger, U. Briel, W. Burkert, K. Dennerl, J. Englhauser, R. Gruber, F. Haberl, G. Hartner, G. Hasinger, M. Kürster, E. Pfeffermann, W. Pietsch, P. Predehl, C. Rosso, J. H. M. M. Schmitt, J. Trümper, and H. U. Zimmermann, The ROSAT all-sky survey bright source catalogue, Astron. Astrophys. 349, 389 (1999).

[39] D. L. Kaplan, Nearby, thermally emitting neutron stars, AIP Conf. Proc. 968, 129 (2008).

[40] D. L. Kaplan and M. H. van Kerkwijk, Constraining the spin-down of the nearby isolated neutron star RX J0806.44123, and implications for the population of nearby neutron stars, Astrophys. J. 705, 798 (2009).

[41] J. A. Kennea et al., Swift discovery of a new soft gamma repeater, SGR J1745-29, near Sagittarius A*, Astrophys. J. 770, L24 (2013).

[42] K. Mori et al., NuSTAR discovery of a 3.76-second transient magnetar near Sagittarius A*, Astrophys. J. 770, L23 (2013).

[43] Ryan M. Shannon and Simon Johnston, Radio properties of the magnetar near Sagittarius A* from observations with the Australia Telescope Compact Array, Mon. Not. R. Astron. Soc. 435, L29 (2013).

[44] R. P. Eatough et al., A strong magnetic field around the supermassive black hole at the centre of the Galaxy, Nature (London) 501, 391 (2013).

[45] F. Nesti and P. Salucci, The dark matter halo of the Milky Way, AD 2013, J. High Energy Phys. 07 (2013) 016.

[46] M. Portail, C. Wegg, O. Gerhard, and I. Martinez-Valpuesta, Made-to-measure models of the Galactic box/peanut bulge:
Stellar and total mass in the bulge region, Mon. Not. R. Astron. Soc. 448, 713 (2015).

[47] D. Hooper, The density of dark matter in the galactic bulge and implications for indirect detection, Phys. Dark Universe 15, 53 (2017).

[48] T. Lacroix, Dynamical constraints on a dark matter density spike at the Galactic Centre from stellar orbits, Astron. Astrophys. 619, A46 (2018).

[49] F. Aharonian (H.E.S.S. Collaboration), Observations of the Sagittarius Dwarf Galaxy by the H.E.S.S. experiment and search for a dark matter signal, Astropart. Phys. 29, 55 (2008); Erratum, Astropart. Phys. 33, 274(E) (2010).

[50] L. Monaco, M. Bellazzini, F. R. Ferraro, and E. Pancino, The central density cusp of the Sagittarius Dwarf Spheroidal Galaxy, Mon. Not. R. Astron. Soc. 356, 1396 (2005).

[51] R. Ibata, M. Bellazzini, S. C. Chapman, E. Dalessandro, F. Ferraro, M. Irwin, B. Lanzoni, G. F. Lewis, A. D. Mackey, P. Miocchi, and A. Varghese, Density and kinematic cusps in M54 at the heart of the Sagittarius Dwarf Galaxy: Evidence for a $10^{4} \mathrm{M}_{\text {sun }}$ black hole?, Astrophys. J. Lett. 699, L169 (2009).

[52] N. Ivanova, C. Heinke, F. A. Rasio, K. Belczynski, and J. Fregeau, Formation and evolution of compact binaries in globular clusters: II. Binaries with neutron stars, Mon. Not. R. Astron. Soc. 386, 553 (2008).

[53] B. R. Safdi, Z. Sun, and A. Y. Chen, Detecting axion dark matter with radio lines from neutron star populations, arXiv:1811.01020.

[54] A. A. Philippov and A. Spitkovsky, Ab-initio pulsar magnetosphere: Three-dimensional particle-in-cell simulations of axisymmetric pulsars, Astrophys. J. 785, L33 (2014).

[55] F. P. Huang, K. Kadota, T. Sekiguchi, and H. Tashiro, The radio telescope search for the resonant conversion of cold dark matter axions from the magnetized astrophysical sources, Phys. Rev. D 97, 123001 (2018). 